\title{
Exposición a la Contaminación con Plomo en Taller de Ensamble de Baterías
}

\author{
EXPOSURE TO LEAD CONTAMINATION IN BATTERY ASSEMBLY WORKSHOP
}

\section{Maria Cristina Bilotta ${ }^{1}$, Purificación Merodo ${ }^{2}$, Aníbal Godoy Ortiz ${ }^{3}$}

1. Médica (Universidad de Buenos Aires, Argentina), Magister en Salud Pública (Universidad del Salvador, Argentina), actual Directora de la Investigación "Patología laboral relacionada con el plomo en taller de baterias", Escuela Superior Técnica (EST), Ejército Argentino.

2. Ingeniera química e ingeniera sanitaria. Actual Directora y profesora de la Maestria en Higiene y Seguridad Ocupacional, Escuela Superior Técnica (EST), Ejército Argentino.

3. Profesor e investigador de la Maestría en Higiene y Seguridad Ocupacional, Escuela Superior Técnica (EST), Ejército Argentino. Candidato a Magíster en Economía de la Salud y Gestión de Organizaciones de Salud (Universidad Nacional de La Plata, Argentina).

\section{RESUMEN}

Introducción: Las consecuencias perjudiciales del plomo $(\mathrm{Pb})$ en la salud de las personas, y los trabajadores en particular, han sido suficientemente demostradas desde hace mucho tiempo. No obstante ser un problema de tan larga data, llega hasta la actualidad; y los talleres de baterías constituyen una de las fuentes de contaminación. Objetivo General: Conocer el nivel de exposición al $\mathrm{Pb}$ de la totalidad de trabajadores directos de un taller de ensamble de baterías de la localidad de Boulogne, Buenos Aires. Objetivos Específicos: -Evaluar los registros sobre el nivel de $\mathrm{Pb}$ en sangre de los trabajadores entre 2007 y 2013. -Describir las condiciones de trabajo y las prácticas higiénicas de los empleados. -Medir la cantidad de $\mathrm{Pb}$ existente en el aire del establecimiento. Material y métodos: Es un estudio observacional descriptivo. El relevamiento general de riesgos se realizó mediante observación directa. Se analizaron las plombemias de los trabajadores. Las prácticas higiénicolaborales se estudiaron mediante un cuestionario semi-estructurado. Se llevó a cabo un estudio del aire. Resultados: El cumplimiento de las normas de seguridad e higiene laboral es parcial. Es una planta pequeña y mal ventilada, sin un adecuado sistema de extracción ni ventilación. No utiliza proceso húmedo o con aspiradores para la limpieza. No es sistemático el recambio de filtros de la campana de extracción ni de los respiradores personales. Las plombemias realizadas entre diciembre de 2007 y marzo de 2013 muestran valores superiores a los límites aceptables, alcanzando el promedio 19,23 ug/100ml (rango: $3 \mathrm{ug} / 100 \mathrm{ml}$ - 48,8 $\mathrm{ug} / 100 \mathrm{ml}$ ). Existen debilidades en cuanto a la utilización de la máscara de protección con filtros, a su higienización y a la forma en que esto se realiza. Resultados de las muestras del aire: en crisol: concentración de plomo (resultado analítico): 0,06 mg/ $/ \mathrm{m}^{3}$; en intercelda: 0,92 mg/m3; en el sector de etiquetado: $0,03 \mathrm{mg} / \mathrm{m}^{3}$. Conclusiones principales: Se requiere el mejoramiento de las normas de seguridad e higiene laborales.

(Bilotta M, Merodo P, Godoy A, 2013. Exposición a la Contaminación con Plomo en Taller de Ensamble de Baterías. Cienc Trab. Sep-Dic; 15 [48]: 158-164).

Palabras clave: CONTAMINACIÓN PLÚMBICA; EXPOSICIÓN LABORAL; FÁBRICA DE BATERÍAS.

\section{ABSTRACT}

Introduction: The harmful effects of lead $(\mathrm{Pb})$ on the health of people and workers in particular have long been sufficiently demonstrated. However being a problem as long-standing, arrives to present workshops and batteries are one of the sources of pollution. General Objective: To determine the level of exposure to $\mathrm{Pb}$ of all direct workers of a battery assembly workshop in the town of Boulogne, Buenos Aires. Specific Objectives: -Evaluate the records on the level of $\mathrm{Pb}$ in blood of workers between 2007 and 2013. -Describe the working conditions and hygiene practices of employees. -Measure the amount of $\mathrm{Pb}$ in the air existing in the establishment.Material and Methods: It is an observational descriptive study. The general risk survey-stage was conducted by direct observation. The lead levels of workers were analyzed. Hygiene and work practices were studied using a semi-structured questionnaire. It was made an air study. Results: Compliance with safety and hygiene is partial. It is a small and stuffy plant without adequate ventilation or exhaust system. It does not use wet or vacuum for cleaning process. No systematic replacement filters range hood or personal respirators. The lead levels between December 2007 and March 2013 show above acceptable limits values, reaching 19,23 ug/100ml average (range: $3 \mathrm{ug} / 100 \mathrm{ml}$ - 48,8 ug/100ml). There are weaknesses with regard to the use of the protective mask filters, to sanitize and the way this is done. Results of air samples: in pot: lead concentration (analytical result): $0,06 \mathrm{mg} /$ $\mathrm{m} 3$, in intercell $0,92 \mathrm{mg} / \mathrm{m} 3$; labeling sector: $0,03 \mathrm{mg} / \mathrm{m} 3$. Main conclusions: improving the safety and health at work is required.

Keywords: LEAD CONTAMINATION, OCCUPATIONAL EXPOSURE, BATTERY FACTORY.

\section{Correspondencia / Correspondence:}

Escuela Superior Técnica (EST) "Gral. Div. Manuel N. Savio" del Ejército Argentino

Av. Cabildo 15, Ciudad Autónoma de Buenos Aires, Argentina

e-mail: cristinabilotta@yahoo.com.ar; purimerodo@yahoo.com.

ar;godoyortiz_anibal@yahoo.com.ar

Tel. y fax EST: (54-11) 4779-3318

Recibido: 16 de Ocubre 2013 / Aceptado 28 de Noviembre 2013

\section{INTRODUCCIÓN}

El plomo $(\mathrm{Pb})$ es un metal pesado ampliamente utilizado en la industria, cuyos efectos tóxicos sobre el organismo humano están ampliamente demostrados.

Las primeras evidencias sobre intoxicación con $\mathrm{Pb}$ pueden encontrarse en períodos muy anteriores a la Revolución Industrial ${ }^{1}$, pero la descripción de los efectos perjudiciales de este metal sobre 
trabajadores y niños se realizó por primera vez en el siglo XIX. Los primeros estudios que mostraron el impacto de la acumulación del $\mathrm{Pb}$ en la salud humana, y particularmente en el sistema nervioso, se conocieron hacia $1920 .^{2}$

La enfermedad ambiental y profesional provocada por este tóxico se denominó "saturnismo". La incorporación al organismo se da fundamentalmente a través de la inhalación e ingesta y, en mucha menor medida, a través de la dermis.

Desde hace varias décadas la contaminación con $\mathrm{Pb}$ es una preocupación de la Organización Mundial de la Salud (OMS), la Organización Internacional del Trabajo (OIT), la Organización de las Naciones Unidas (ONU) y de todos los responsables de la salud de los trabajadores.

Entre las fuentes de contaminación se pueden mencionar la industria minera, las fábricas de construcción y reacondicionamiento de baterías; las de acumuladores para automotores, las plantas de soldadura, las imprentas, los basurales a cielo abierto, la industria del cemento, de pinturas, barnices y esmaltes; las de anzuelos con $\mathrm{Pb}$ para la pesca, las plantas térmicas, entre otros. Corresponde destacar que el trabajador puede transportar el $\mathrm{Pb}$ en su ropa al hogar, afectando de este modo a su familia, fenómeno incluido bajo la denominación de "domiciliarización de los riesgos ocupacionales".

Obviamente la exposición y el consecuente riesgo de contaminación son más elevados en el sector informal de la economía, donde se da un mayor incumplimiento de las normas de seguridad e higiene laboral y ambiental vigentes.

La absorción del $\mathrm{Pb}$ en expuestos depende de múltiples factores.

Es mayor:

- Cuanto más pequeña es la partícula. El modelo formulado por Bernard (1977) e integrado por Ashford et al (1977) estimó una absorción del 37\% para las partículas de $\mathrm{Pb}$ en aire menores a $1 \mathrm{um}$, mientras que para las mayores a ese tamaño la absorción prevista fue de 1,8\%. (3) Sin embargo, se ha sostenido que el porcentaje de absorción de polvos comprendidos entre 1 y 5 um y entre 5 y 10 um puede superar el $8 \%$. $^{3}$

- Cuanto mayor es la solubilidad: algunas sales de Pb son absorbidas en menor cuantidad debido a su baja solubilidad. ${ }^{3}$

- Cuanto mayor sea el tiempo de exposición.

Y si la persona presenta:

- diabetes o hipertensión, que pueden incrementar la nefro-toxicidad del $\mathrm{Pb}^{4}$;

- enfermedad renal crónica ${ }^{5}$;

- tabaquismo: el hábito de fumar en personas con niveles moderados $\mathrm{de} \mathrm{Pb}$ en sangre está asociado a mayor mortalidad por cáncer ${ }^{6}$;

- consumo de alcohol en exceso.

El daño también puede ser mayor debido a:

- la deficiencia de hierro o zinc ${ }^{7}$;

- si la ingesta de grasas es elevada. Y la absorción de Pb es mayor con la ingesta de ácidos grasos polinsaturados que con los satu$\operatorname{rados}^{7}$;

- si el estado nutricional no es adecuado;

- si el estómago está vacío y

- si se es niño: en la población infantil la absorción de Pb es del 30 al 50\%, mientras que en el adulto es del 10\%. ${ }^{8}$

Cada vez es mayor la acumulación de conocimientos científicos sobre los efectos adversos que provoca este metal y sus derivados.
Y a la luz del avance de la ciencia los valores que se recomiendan como límites admisibles de exposición son cada vez más bajos. ${ }^{9}$ Incluso los estándares establecidos en algunos países, Estados Unidos en particular, vienen siendo cuestionados desde hace más de 20 años por existir toxicidad a niveles inferiores a los normados. ${ }^{1,6,10}$ También en países como Italia ${ }^{11} \mathrm{y}$ Brasil ${ }^{12}$ se ha solicitado la revisión integral de la legislación en la materia.

En la literatura científica se registran pocos casos de intoxicación aguda en la industria, tales como los observados por Apostoli en Italia. ${ }^{13}$ En cuanto a la exposición crónica, es bien sabido que provoca severos e irreversibles daños a la salud humana.

Compromete, entre otros:

- el sistema nervioso central y periférico,

- las gónadas,

- el funcionamiento renal: la toxicidad por $\mathrm{Pb}$ resultante de la exposición durante largo tiempo a niveles relativamente elevados suele manifestarse por medio de nefropatía crónica y puede conducir al fallo renal. En el mismo sentido, diversos estudios epidemiológicos observaron exceso de mortalidad por enfermedad renal en trabajadores expuestos. ${ }^{14}$ También se han identificado efectos renales adversos con promedios de $\mathrm{Pb}$ en sangre de $<5 \mathrm{ug} / \mathrm{dl} .^{5}$ No obstante, para hallazgos contradictorios e inexistencia de daño renal después de muchos años de exposición al $\mathrm{Pb}$ entre moderada y fuerte en establecimientos fabriles de Suecia y Bélgica ver Gerhardsson et al. 1992 y Roels et al. 1994, respectivamente ${ }^{15,16}$;

- altera la médula ósea y los huesos, los cuales le sirven como depósito durante largos períodos de tiempo (años o aún décadas): la vida media del $\mathrm{Pb}$ en hueso estable oscila entre 10 y 20 años. ${ }^{17} \mathrm{El} \mathrm{Pb}$ acumulado en el hueso representa el 95\% de la carga total de $\mathrm{Pb}$ del organismo y puede constituirse en una fuente endógena de contaminación cuando el mismo es movilizado en circunstancias tales como el embarazo, la lactancia, la menopausia o el envejecimiento ${ }^{5,18}$;

- también afecta al sistema cardiovascular ${ }^{18}$, esencialmente por la relación causal existente entre exposición al $\mathrm{Pb}$ e hipertensión (HTA): un incremento de $5 \mathrm{ug} / \mathrm{dl}$ de $\mathrm{Pb}$ en sangre está asociado con un aumento en la presión arterial sistólica de entre 0,6 a 1,25 mmHg. ${ }^{6,10}$ Además de la HTA y el daño renal, el $\mathrm{Pb}$ compromete la función arterial a través del estrés oxidativo y la disfunción endotelial. ${ }^{19}$ Finalmente, Khalil et al observaron un incremento de mortalidad en mujeres con niveles de $\mathrm{Pb}$ en sangre $>8 \mathrm{ug} / \mathrm{dl}$, en particular por enfermedad cardio-coronaria, en comparación con aquellas que presentaron valores de plombemia inferiores.

El $\mathrm{Pb}$ disminuye la capacidad de aprendizaje y cognitiva en los niños por el daño neurológico que ocasiona. En adultos, el impacto neuro-comportamental de la exposición baja a moderada es más controversial y son escasos los estudios que analizan este efecto de la exposición acumulada en el largo plazo. ${ }^{20}$ En las mujeres, por traspasar la barrera placentaria, puede provocar abortos espontáneos.

$\mathrm{El} \mathrm{Pb}$ inhibe la habilidad del cuerpo para fabricar hemoglobina, al interferir con varios pasos enzimáticos de la ruta del grupo hemo. Por ello, el plomo puede provocar dos tipos de anemia, comúnmente acompañadas de punteado basófilo de los eritrocitos. También impide la conversión de la vitamina $\mathrm{D}$, responsable del mantenimiento de la homeostasis del calcio extracelular e intracelular. Así, el $\mathrm{Pb}$, al inhibir o mimetizar las acciones del calcio, 
puede dificultar el crecimiento celular, la maduración y el desarrollo de huesos y dientes.

La contaminación plúmbica se manifiesta de diversas formas y fundamentalmente por medio de sintomas inespecíficos que dificultan la realización del diagnóstico médico precoz: hipertensión arterial (ya mencionada), anemia, cansancio crónico, disminución de la libido, esterilidad tanto en hombres como en mujeres; trastornos del sueño, irritabilidad, cefalea, problemas digestivos (síndrome doloroso abdominal paroxístico afebril), calambres, mialgias y artralgias, poliuria, nicturia, gota, debilidad en los dedos, las muñecas (fenómeno conocido como la "mano del pintor") o los tobillos.

El sintoma más específico de la exposición a este metal es el denominado "ribete de Burton", que consiste en una línea oscura que se forma entre la base del diente y la encía debido a que el sulfuro liberado por las bacterias se une al plomo. Por este motivo es tan importante, como una de las tantas medidas preventivas, la adecuada higiene oral.

En la actualidad no existe evidencia concluyente sobre el efecto carcinógeno del $\mathrm{Pb}$ en humanos. No obstante, la Agencia Internacional para la Investigación del Cáncer (IARC, por sus siglas en inglés), organismo perteneciente a la OMS, primero incluyó al $\mathrm{Pb}$ y sus compuestos inorgánicos en el Grupo $2 \mathrm{~B}$ (posible cancerígeno para el hombre) y luego de una actualización en el Grupo 2 A (probable cancerígeno para humanos). ${ }^{21}$

Entre las recomendaciones de la OIT y otros organismos para la prevención de la contaminación plúmbica en trabajadores expuestos se mencionan las siguientes:

- Reducir al mínimo indispensable la utilización del Pb. En este caso, esto sólo sería posible con el cambio del tipo de baterías y la consecuente modificación de los vehículos para los cuales están destinados.

- Preferir, en la medida que sea posible, ropa de protección de fibras sintéticas, ya que éstas retienen menos polvo que las de algodón.

- Evitar la ropa con pliegues y/o bolsillos, los cuales favorecen la retención del polvo.

- Lavar o cambiar la ropa regularmente.

- Disponer de armarios especiales para los elementos de protección personal y separados de la ropa de calle.

- Proveer duchas con agua caliente.

- No llevar nunca la ropa de trabajo al hogar.

- Dar a los trabajadores el tiempo necesario para asearse antes de comer.

- Prohibir comer, ingerir bebidas o fumar en las áreas cercanas a las zonas de procesamiento de plomo.

- Establecer zonas especialmente acondicionadas para comer o ingerir bebidas.

- Mantener una adecuada y permanente limpieza de las salas y del edificio donde se trabaja con $\mathrm{Pb}$ mediante un proceso húmedo o con aspiradores. No barrer, ya que esto remueve el polvo.

- Proporcionar protección respiratoria adecuadamente utilizada y mantenida para asegurar su eficacia. La barba o el vello facial atentan contra la adherencia del respirador. Se debe recordar finalmente que un respirador con filtros en mal estado o los barbijos favorecen la absorción pulmonar del $\mathrm{Pb}$.

Es importante aclarar que una sola medida de protección no implementada, o mal realizada, puede dar lugar a la contaminación plúmbica. Y la concreción de algunas de estas prácticas de ningún modo sustituye a las otras.
Valores de referencia para población no expuesta

Para la población adulta sana no expuesta laboralmente al $\mathrm{Pb}$ los valores de referencia para el $\mathrm{Pb}$ en sangre son los siguientes ${ }^{22}$ :

- mujeres 2,0 a $15,2 \mathrm{ug} / 100 \mathrm{ml}$

- hombres 3,1 a $17,7 \mathrm{ug} / 100 \mathrm{ml}$

Para los niños el valor límite es $10,0 \mathrm{ug} / 100 \mathrm{ml} .{ }^{23}$ Por lo expuesto anteriormente, consideramos que en este caso no existe un nivel de exposición seguro.

Es importante destacar que en la Argentina el nivel de $\mathrm{Pb}$ en sangre en no expuestos laboralmente viene disminuyendo como consecuencia de la supresión, en el año 1997, de este metal en las naftas; el reemplazo de cañerías domiciliarias de $\mathrm{Pb}$ por otras plásticas y la sustitución de soldaduras de $\mathrm{Pb}$ por las eléctricas en las latas de conservas alimenticias. ${ }^{17}$ Todas las plombemias del "grupo control" realizadas en mayo de 2013 (en el marco de esta investigación) a 10 personas no expuestas y de similares características a las de los trabajadores, fueron inferiores a 5,0 ug/100 ml.

\section{Valores y controles para personas expuestas laboralmente}

Los exámenes periódicos a realizar en los trabajadores expuestos son $^{24}$ :

Anualmente:

- Examen clínico general con orientación neurológica y cardiovascular.

- Hemograma.

- Orina: beta-2-microglobulina.

- Urea sanguínea.

- Uricemia.

- Creatinina plasmática.

Semestralmente:

- Plombemia $(\mathrm{Pb})$ o Protoporfirina eritrocitaria (PPE) y ácido delta-amino-levulínico en orina (ALA-U).

- Protoporfirina libre. Valor de referencia: < $75 \mathrm{mcg} / 100 \mathrm{ml} \mathrm{de}$ hematíes. Índice Biológico de Exposición: hasta $300 \mathrm{mcg} / 100$ ml de hematíes.

- Ácido delta-amino-levulínico. Valor de referencia: < a 4,5 mg/g de creatinina. Índice Biológico de Exposición: $10 \mathrm{mg} / \mathrm{g}$ de creatinina.

Para las personas expuestas laboralmente el valor límite de $\mathrm{Pb}$ en sangre (plombemia) establecido en la Argentina es: $30 \mathrm{ug} / 100 \mathrm{ml}$. Mientras no se alcance este valor, y/o el ácido delta-aminolevulínico en orina (ALA-U) sea $<$ a $10 \mathrm{mg} / \mathrm{g}$ de creatinina o la Protoporfirina eritrocitaria (PPE) sea < a $300 \mathrm{mcg} / 100 \mathrm{ml}$ corresponde la realización de estudios semestrales. ${ }^{24}$

Alcanzado el valor límite de $\mathrm{Pb}$ en sangre, las acciones que se deben realizar son las siguientes ${ }^{24}$ :

Con una plombemia entre 30 y $60 \mathrm{ug} / 100 \mathrm{~mL}$, y/o ALA-U de 10 a $15 \mathrm{mg} / \mathrm{g}$ de creatinina o PPE > a $300 \mathrm{mcg} / 100 \mathrm{ml}$ :

- Evaluación del ambiente laboral y corrección de falencias.

- Realización de control trimestral del trabajador hasta normalizar los valores.

- Formación del trabajador en cuanto a normas de seguridad e higiene.

- Restablecer el control semestral.

Con una plombemia mayor de 60 y hasta $80 \mathrm{ug} / 100 \mathrm{~mL}, \mathrm{y} / \mathrm{o}$ ALA-U > a $15 \mathrm{mg} / \mathrm{g}$. de creatinina, o PPE > a $300 \mathrm{mcg} / 100 \mathrm{ml}$, con o sin manifestaciones clínicas (tales como cefalea, disminución de la libido, trastornos digestivos, cansancio, etc.): 
- Revisión del medio ambiente laboral y de las prácticas de seguridad e higiene.

- Retirar al trabajador.

- Implementar tratamiento, si corresponde.

- Controlar al trabajador en 15 días.

- Luego del retorno al trabajo, se hará control trimestral durante seis meses.

- Educar al trabajador.

- Restablecer el control semestral.

Con una plombemia > a $80 \mu \mathrm{g} / 100 \mathrm{ml}$ y/o ALA-U > a $15 \mathrm{mg} / \mathrm{g}$ de creatinina, o PPE > a $300 \mu \mathrm{g} / 100 \mathrm{ml}$, con o sin algunas de las siguientes manifestaciones de enfermedad reversibles: cansancio fácil, cefaleas, hipersomnia, trastornos digestivos (especialmente dolor abdominal), disminución de la libido, modificaciones en el espermograma, etc.:

- Establecer tratamiento médico.

- Revisión del medio ambiente laboral y de las prácticas de seguridad e higiene.

- Control clínico y de laboratorio a los 10 días de finalizado el tratamiento. De acuerdo a dicha evaluación decidir el retorno a la exposición.

- Una vez normalizados los valores y reintegrado al trabajo, control trimestral durante seis meses.

- Luego, retomar el control semestral.

Con una plombemia mayor a $80 \mathrm{ug} / 100 \mathrm{~mL}, \mathrm{y} / \mathrm{o}$ ALA-U > a $15 \mathrm{mg} / \mathrm{g}$ de creatinina, o PPE > a $300 \mu \mathrm{g} / 100 \mathrm{ml}$, con las siguientes manifestaciones de enfermedad: anemia, compromiso renal, manifestaciones de encefalopatía, compromiso neurológico periférico clínico-electromiográfico:

- Retirar al trabajador.

- Determinar el tratamiento médico.

- Control clínico-neurológico, control electromiográfico, si fuere necesario, hasta mejoría y control de laboratorio.

- Implementar medidas ambientales.

- Educación del trabajador.

- Recalificar, si corresponde; y control trimestral durante un año.

\section{MATERIAL Y MÉTODOS}

Se analizaron los registros de plombemia realizados desde diciembre de 2007 hasta marzo de 2013. El nivel de Pb en sangre es un indicador de exposición reciente, no de la carga total de $\mathrm{Pb}$ en el organismo. Esto último requiere el estudio de la cantidad de este metal depositado en el hueso, a través de rayos x fluorecentes. También la zincprotoporfirina eritrocitaria (ZPP) fue sugerida como indicador de exposición acumulada en los 120 días previos a la medición. ${ }^{11}$ Otra alternativa para medir lo acumulado es construir un índice que sume el valor promedio anual $\mathrm{de} \mathrm{Pb}$ en sangre de cada trabajador en umol/l como indicador de exposición previa más preciso que la duración del empleo. ${ }^{15}$

Desde diciembre de 2007 hasta abril de 2011 inclusive, las plombemias fueron realizadas por una institución privada (Laboratorio Córdoba), siendo la metodología utilizada absorción atómica - $\mathrm{h}$. grafito. A partir de mayo de 2011 los estudios fueron hechos por la Facultad de Farmacia y Bioquímica, Cátedra de Toxicología y Química Legal de la Universidad de Buenos Aires (UBA). La metodología utilizada fue la misma: espectofotometría de absorción atómica, atomización electrotérmica.
Para el estudio de las prácticas higiénico-laborales se diseñó un cuestionario semi-estructurado, con preguntas abiertas y cerradas, completados en forma anónima por cada uno de los empleados.

La medición ambiental fue realizada por la empresa Laia. Se efectuó la toma de muestra del aire en tres puestos de la línea de producción (crisol, intercelda y etiquetado). Se utilizó como instrumental Rotámetro DWYER, Bomba SKC, Espectrómetro de absorción Atómica, marca Varian Modelo 55B de serie EL020 35769.

\section{RESULTADOS}

\section{De la observación in situ:}

La investigación se realizó en un taller que consta de diez puestos de trabajo: Puesto 1: Colada (armado de piezas); 2: Ajuste (limado y ensobrado de placas); 3: Soldadura (soldadura de vasos y armado de paquetes); 4: Armado (armado de baterías); 5: Interceldas (soldadura de interceldas); 6: Termosellado (sellado de cajas); 7: Control (marcado de bornes); 8: Carga (carga de baterías); 9: Control final (prueba de carga y descarga); 10: Embalaje (armado de caja, embalaje y cierre).

\section{Gráfico 1.}

Esquema de la planta fabril.
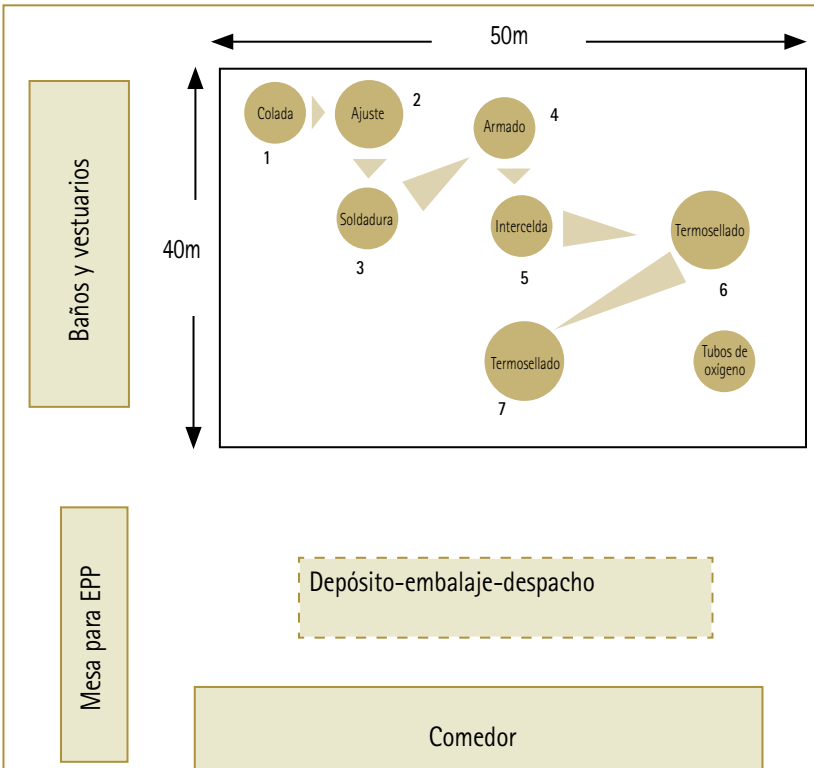

Comedor

Se observan como principales debilidades las siguientes:

- La planta no cuenta con un adecuado sistema de extracción ni ventilación. Carecen de un procedimiento sistematizado de recambio de filtros de la campana de extracción y, además, éstos son de fabricación casera y de efectividad desconocida. Tampoco se mide el caudal de aire aspirado.

- No utiliza proceso húmedo o con aspiradores para la limpieza, sino que se barre.

- El ambiente laboral no es libre de humo proveniente del tabaco y existe en el interior del establecimiento un contenedor específico para arrojar las colillas del cigarrillo.

- Los trabajadores disponen de respiradores faciales de media cara (Series 6000 de la marca 3M) y los correspondientes cartuchos 
Tabla 1.

Plomo en sangre (ug/100 $\mathrm{ml}$ ).

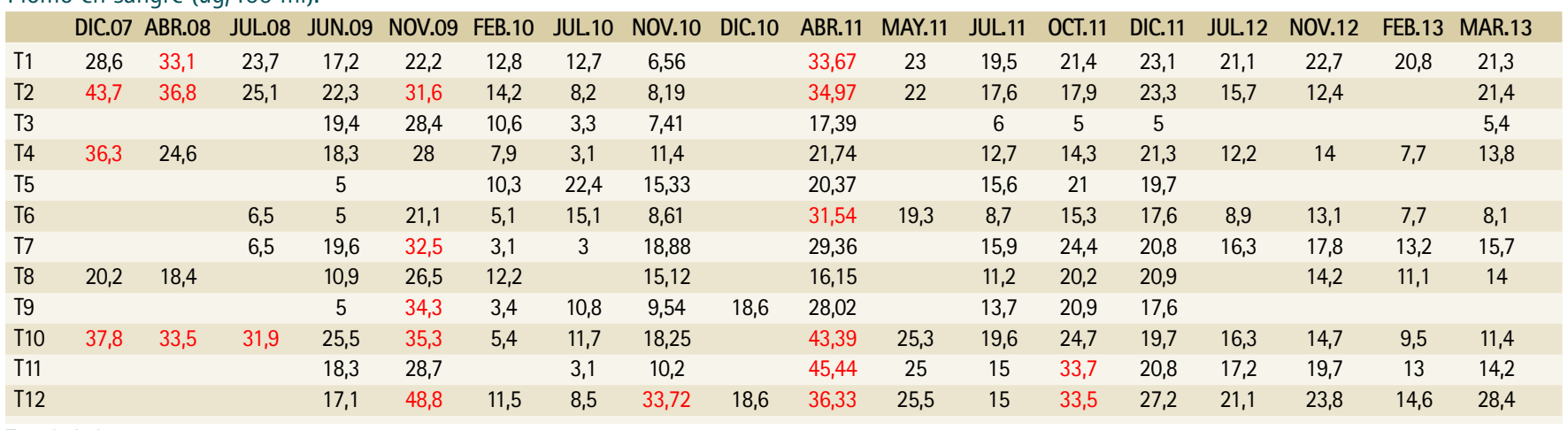

T: trabajador

contra vapores orgánicos/gases. No obstante, no está sistematizado el recambio de estos últimos según las especificaciones del fabricante (deben reemplazarse si se dañan o luego de 40 horas de uso o 30 dias, lo que ocurra primero) y dicha reposición muchas veces se ve impedida debido a faltantes de stock.

- El sector comedor o área de descanso se encuentra en el mismo ambiente de trabajo y no cuenta con las barreras de protección adecuadas.

De los registros de plombemias:

El promedio de $\mathrm{Pb}$ en sangre para todos los trabajadores es 19,23 $\mathrm{ug} / 100 \mathrm{ml}$. Se detectaron plombemias individuales que llegaron hasta 48,8 ug/100 ml en noviembre de 2009. Los promedios más altos se registraron en los meses de: diciembre de 2007 (33,3 ug/100 ml), abril de $2008(29,3)$, noviembre de $2009(30,7)$ y abril de $2011(29,9)$.

De la encuesta:

Todos los trabajadores encuestados $(n=9)$ son hombres. El $66,67 \%$ (6) tienen entre 21 y 25 años de edad, el 22,2\% (2) entre 26 y 30 , y el 11,11\% (1) entre 61 y 65 años. En cuanto al nivel de instrucción alcanzado, el 44,44\% (4) completó el secundario, el $44,44 \%$ (4) tiene el secundario incompleto, y el 11,1\% (1) posee nivel universitario incompleto. Otros antecedentes de importancia son: el 77,78\% (7) tuvo un trabajo anterior al actual, de los cuales 2 pueden ser considerados expuestos al plomo (construcciónpinturas y construcción). Dos empleados (22,22\%) tienen otro trabajo además del actual: cadete y operario en un taller particular. Finalmente 7 (77,78\%) recibieron capacitación específica para la función actual. El promedio de antigüedad en el puesto es de 4 años (rango: 2 a 6).

Se identifican principalmente falencias en cuanto a las máscaras de protección con filtros (ver Tabla 2).

De la medición ambiental:

La Concentración Máxima Permisible (CMP) para el $\mathrm{Pb}$ en aire, según lo establecido por la Resolución 295/2003 del Ministerio de Trabajo, Empleo y Seguridad Social de la República Argentina, es $0,05 \mathrm{mg} / \mathrm{m} 3$. Este valor está establecido para una jornada de 8 horas diarias y 40 horas semanales.

En este taller la jornada laboral es de 4 horas, 4 días a la semana, total 16 horas semanales.

Método o norma utilizada en la medición: NIOSH7082, límite de cuantificación del método: $0,01 \mathrm{mg} / \mathrm{m} 3$.
Tabla 2.

Hábitos higiénico-laborales de los obreros expuestos al plomo $(n=9)$.

$\begin{array}{lcccc}\text { Práctica } & \begin{array}{c}n(\%) \\ \text { Siempre }\end{array} & \begin{array}{c}n(\%) \\ A \text { veces }\end{array} & \begin{array}{c}n(\%) \\ \text { Nunca }\end{array} & \text { Total } \\ \text { Utiliza indumentaria de trabajo } & 9(100) & 0(0) & 0(0) & 9(100) \\ \text { Utiliza mameluco externo para ingreso a } & & & & \\ \text { puestos de trabajo } & 6(66,67) & 2(22,22) & 1(11,11) & 9(100) \\ \text { Utiliza zapatos de seguridad } & 9(100) & 0(0) & 0(0) & 9(100) \\ \text { Utiliza máscara de protección con filtros } & 8(88,89) & 1(11,11) & 0(0) & 9(100) \\ \text { Higieniza la máscara de protección con filtros } & 4(44,44) & 5(55,56) & 0(0) & 9(100) \\ \text { Utiliza guantes para trabajar } & 9(100) & 0(0) & 0(0) & 9(100) \\ \text { Ingiere algún tipo de alimentos o bebidas } & & & & \\ \text { en los puestos de trabajo } & 0(0) & 0(0) & 9(100) & 9(100) \\ \text { Fuma en el ámbito de trabajo } & 0(0) & 0(0) & 9(100) & 9(100)\end{array}$

Fuente: Elaboración propia a partir de encuesta anónima realizada en mayo de 2012.

Lugar 1: Crisol

- Analito: PLOMO

- Concentración (resultado analítico) 0,06 mg/m3

Lugar 2: Intercelda

- Analito: PLOMO

- Concentración (resultado analítico) 0,92 mg/m3

Lugar 3: Sector de etiquetado

- Analito: PLOMO

- Concentración (resultado analítico) 0,03 mg/m3

\section{DISCUSIÓN}

En este trabajo se incluyeron datos preliminares de una investigación más amplia que concluirá en el año 2013 y que incluirá otros establecimientos. Como aspecto destacable se menciona la utilización de diversas fuentes de información: observación directa, mediciones de plomo en sangre y ambiental, y encuesta sobre prácticas laborales.

Es evidente que en esta planta fabril, si bien se observa el esfuerzo del cumplimiento de ciertas normas de seguridad e higiene, no existe rigurosidad en las prácticas laborales preventivas.

Los niveles de $\mathrm{Pb}$ en sangre de los trabajadores en muchos casos superan los limites permitidos, que están establecidos en la legislación para el doble de horas diarias a las efectivamente realizadas en esta planta. Es sabido que con la implementación de las medidas necesarias dichos valores se verían reducidos sustancialmente. 
Finalmente, por ser cada vez mayor la evidencia que muestra la existencia de toxicidad y morbi-mortalidad asociados a valores inferiores a los establecidos legalmente, consideramos legítimo discutir la conveniencia de establecer estándares más bajos, esto sin descuidar su factibilidad económica.

\section{AGRADECIMIENTOS}

Los autores agradecen al Dr. Raúl de Tito y a la Dra. Marta Cristina Biagi por la lectura de versiones preliminares de este trabajo; así como también al Lic. Martín Wesenack por su cola- boración en el análisis estadístico. La responsabilidad es exclusiva de los autores.

Institución donde se realizó la investigación

Este artículo expone los hallazgos parciales de una investigación más amplia que se está llevando a cabo desde marzo de 2012 en el marco de la investigación "Patología laboral relacionada con el plomo en taller de baterías", Escuela Superior Técnica (EST), Ejército Argentino. Su finalización está prevista para diciembre de 2013.

\section{Fuente de financiamiento}

Esta investigación se está realizando con los recursos proporcionados por la Escuela Superior Técnica (EST), institución universitaria perteneciente al Ejército Argentino. No existen conflictos de interés.

\section{REFERENCIAS}

1. Landrigan PJ. Lead in the modern workplace. Am J Public Health [en linea].1990 [consultado 11 oct 2012]; 80:907-908. Disponible en: http://www. ncbi.nlm.nih.gov/pmc/articles/PMC1404786/pdf/amjph00221-0009.pdf

2. Ibels LS, Pollock CA. Lead intoxication. Med Toxicol. 1986;1:387-410.

3. Apostoli P. Aggiornamenti in tema di tossicologia da piombo. Ann Ist Sup Sanità [en línea]. 1998 [consultado 30 abr 2013];34:5-15. Disponible en: http://www. eatris.it/binary/publ/cont/Pag._5_15_Annali_Vol._34_N._1_1998.pdf

4. Tsaih SW, Korrick S, Schwartz J, Amarasiriwardena C, Aro A, Sparrow D, et al. Lead, diabetes, hypertension, and renal function: the Normative Aging Study. Environ Health Perspect [en línea]. 2004; [consultado 15 oct 2012];112:1178- 1182. Disponible en: http://www.ncbi.nlm.nih.gov/pmc/ articles/PMC1247478/

5. Ekong EB, Jaar BG, Weaver VM: Lead-related nephrotoxicity: a review of the epidemiologic evidence. Kidney Int 2006;70: 2074-2084.

6. Lustberg M, Silbergeld E. Blood lead levels and mortality. Arch Intern Med 2002; $162: 2443-2449$

7. U.S. Environmental Protection Agency. Air Quality Criteria for Lead; 2006 Final Report. [en línea].Washington: EPA; 2006. [consultado 8 nov 2012]. (EPA/600/R-05/144aF-bF). Disponible en: http://cfpub.epa.gov/ncea/cfm/ recordisplay.cfm?deid=158823\#Download.

8. Valdivia Infantas MM. Intoxicación por plomo. Rev Soc Per Med Inter [en linea]. 2005 [consultado 11 oct 2012]; 18(1):22-7. Disponible en: http:// sisbib.unmsm.edu.pe/BVrevistas/spmi/v18n1/pdf/a05v18n1.pdf

9. Sanin LH, Gonzalez-Cossio T, Romieu I, Peterson KE, Ruiz S, Palazuelos E, et al. Effect of maternal lead burden on infant weight and weight gain at one month of age among breast-fed infants. Pediatrics. 2001; 107:1015-1023.

10. Navas-Acien A, Guallar E, Silbergeld EK, Rothenberg SJ. Lead exposure and cardiovascular disease - a systematic review. Environ Health Perspect. 2007; 15:472-482.

11. Apostoli P, Alessio L. II Decreto Legislativo 25/2002 garantisce ancora la tutela della salute dei lavoratori esposti a piombo?. G Ital Med Lav Erg [en línea]. 2002 [consultado 30 abr 2013]; 24(4):355-360. Disponible en: http://www.gimle.fsm.it/24/4/1.pdf

12. Araujo UC, Pivetta FR, Moreira JC. Avaliação da exposição ocupacional ao chumbo: proposta de uma estratégia de monitoramento para prevenção dos efeitos clínicos e subclínicos. Cad Saúde Pública [en línea]. 1999; [consultado 26 jun 2013];15(1):123-31. Disponible en: http://www.scielo. $\mathrm{br} / \mathrm{pdf} / \% 0 \mathrm{D} / \mathrm{csp} / \mathrm{v} 15 \mathrm{n} 1 / 0042 . \mathrm{pdf}$

13. Apostoli P. Evoluzione dell'esposizione a piombo negli ambienti di lavoro e di vita. Ann Inst Sup Sanità [en línea].1998 [consultado 30 abr 2013]; 34:121-129. Disponible en: http://www.iss.it/binary/publ/cont/Pag._121_ 129_Annali_Vol._34_N._1_1998.pdf

14. Landrigan PJ. Toxicity of lead at low dose (Editorial). Brit J Ind Med [en línea].
1989 [consultado10 oct 2012];46: 593-596. Disponible en: http://www.ncbi. nlm.nih.gov/pmc/articles/PMC1009833/pdf/brjindmed00137 -0001.pdf

15. Gerhardsson L, Chettle DR, Englyst V, Nordberg GF, Nyhlin H, Scott MC et al. Kidney effects in long term exposed lead smelter workers. Brit J Ind Med [en linea]. 1992 [consultado 15 oct 2012]; 49:186-192. Disponible en: http://www. ncbi.nlm.nih.gov/pmc/articles/PMC1012092/pdf/brjindmed 00015-0044.pdf

16. Roels $H_{1}$ Lauwerys $R$, Konings J, Buchet JP, Bernard A, Green S et al. Renal function and hyperfiltration capacity in lead smelter workers with high bone lead. Occup Environ Med [en línea].1994 [consultado 17 oct 2012]; 51(8): 505-512. Disponible en: http://www.ncbi.n/m.nih.gov/pmc/articles/ PMC11280 28/pdf/oenvmed00056-0001.pdf

17. López $C M$, Piñeiro $A E$, Pongelli V, Villamil EC, Roses OE. Valores referenciales de plombemia en el área de Buenos Aires. Población adulta sana no expuesta laboralmente. Acta Bioquim Clín Latinoam [en línea]. 2005 [consultado 25 abr 2013]; 39(4): 453-8. Disponible en: http://www.scielo. org.ar/scielo.php?pid=S0325-29572005000400006\&script=sci_arttext

18. Khalil N, Wilson JW, Talbott EO, Morrow LA, Hochberg MC, Hillier TA et al. Association of blood lead concentrations with mortality in older women: a prospective cohort study. Environ Health [en linea] 2009 [consultado 17 oct 2012]; 8:15. Disponible en: http://www.ehjournal.net/content/pdf/1476069X-8-15.pdf

19. Vaziri ND, Ding $Y, N i Z$. Compensatory up-regulation of nitricoxide synthase isoforms in lead-induced hypertension; reversal by a superoxide dismutase-mimetic drug. J Pharmacol Exp Ther [en línea]. 2001 [Consultado 08 nov 2012]; 298:679-685. Disponible en: http://jpet.aspetjournals.org/ content/298/2/679.full.pdf

20. Balbus-Kornfeld JM; Stewart W; Bolla K I; Schwartz BS. Cumulative exposure to inorganic lead and neurobehavioural test performance in adults: an epidemiological review. Occup Environ Med [en línea].1995 [consultado 10 oct 2012]; 52:2-12. Disponible en: http://www.ncbi.nlm.nih.gov/pmc/articles/PMC1128143/pdf/oenvmed00061-0004.pdf

21. Rousseau MC, Straif K, Siemiatycki J. IARC Carcinogen update. Environ Health Perspect. 2005;113(9): A580.

22. López $C M$, Piñeiro $A E$, Pongelli $V$, Barral de Pizzolato $M C$, Rodríguez $E$, Bassols $G$, et al. Valores referenciales de plombemia en sujetos sanos y no expuestos profesionalmente al plomo. Acta Toxicol Argent. 2003;11(1):33.

23. U.S. Center for Disease Control and Prevention. Managing Elevated Blood Lead Levels Among Young Children: Recommendations from the Advisory Committee on Childhood Lead Poisoning Prevention. Atlanta: CDC; 2002.

24. Albiano, NF. Toxicología laboral. Criterios para la vigilancia de los trabajadores expuestos a sustancias químicas peligrosas [en línea] Buenos Aires: Ministerio de Salud-Superintendencia de Riesgos del Trabajo; 2003. [consultado 11oct 2012]. Disponible en: http://www.msal.gov.ar/redartox/ documentos/TOXICOLOGIALABORAL.pdf 


\section{Referencias complementarias}

1. Argentina. Ministerio de Trabajo, Empleo y Seguridad Social. Higiene y seguridad en el trabajo. Resolución 295/2003 [en línea]. Buenos Aires: Ministerio de Trabajo, Empleo y Seguridad Social; 2003 [consultado el 8 de noviembre de 2012]. Disponible en: http://infoleg.mecon.gov.ar/infoleglnternet/anexos/ 90000-94999/90396/norma.htm

2. De Oliveira Mattos UA, Fortes JDN, da Rocha Shubo AM, Portela LF, Gómez $M B$, Tabalippa $\mathrm{M}$ et al. Avaliação e diagnóstico das condições de trabalho em duas indústrias de baterias chumbo-ácidas no estado do Rio de Janeiro. Ciência \& saude coletiva [en línea]. 2003 [consultado 7 may 2013];8(4): 1047-1056. Disponible en: http://www.scielo.br/pdf/\%0D/csc/ v8n4/a26v8n4.pdf

3. Disalvo L, Aab C, Pereyras S, Pattín J, Apezteguia M, lannicelli JC, et al. Plumbemia en Niños de la Ciudad de La Plata, Argentina y su relación con la deficiencia de hierro y los factores de exposición al plomo. Arch Argent Pediatr[en línea]. 2009 [consultado 23 abr 2013];107(4): 300-6. Disponible en: http://www.scielo.org.ar/scielo.php?pid=S0325-00752009000400005 \&script=sci_arttext\&tIng=pt

4. Gerhardsson L, Lundstrom NG, Nordberg G, Wall S. Mortality and lead exposure: a retrospective cohort study of Swedish smelter workers. $\mathrm{Br} \mathrm{J}$ Ind Med 1986; 43:707-712.

5. Landrigan PJ, Todd AC. Lead poisoning. West J Med [en línea]. 1994 [consultado11 oct 2012]; 161:153-6. Disponible en: http://www.ncbi.nlm. nih.gov/pmc/articles/PMC1022528/pdf/westjmed00060-0047.pdf

6. Martínez Riera N, Soria N, Feldman G, Riera N. Niveles de plombemia y otros marcadores en niños expuestos a una fundición de plomo en Lastenia, Tucumán Argentina. Retel [en línea]. 2003 [consultado $25 \mathrm{abr}$ 2013];11:12-22. Disponible en: http://www.bvsde.paho.org/bvsacd/cd57/ plombemia.pdf

7. Menditto $A$, Chiodo $F$, Patriarca $M$, Morisi G. Esposizione al piombo: Valutazione del rischio per la popolazione generale italiana negli anni 90. Ann Ist Super Sanita [en línea]. 1998 [consultado 30 abr 2013]; 34:27-39. Disponible en: http://www.iss.it/binary/publ/cont/Pag._27_39_Annali_ Vol._34_N._1_1998.pdf

8. Minozzo R, Wagner SC, Santos CH, Deimling LI, Mello RS. Prevalência de anemia em trabalhadores expostos ocupacionalmente ao chumbo. Rev Bras Hematol Hemoter [en línea]. 2009 [consultado 7 may 2013];2(31):94-7. Disponible en: http://www.scielo.br/pdf/rbhh/v31n2/aop1809.pdf

9. Moreno Carrillo A, Granada J. Intoxicación por plomo: diagnóstico diferencial de dolor abdominal crónico. Reporte de caso y revisión de tema. Univ Méd Bogotá Colombia [en línea]. 2012 [consultado 11 oct 2012]; 53(2):199-207. Disponible en: http://med.javeriana.edu.co/publi/vniversitas/serial/v53n2/ Intoxicacion\%20por\%20plomo.pdf

10. Muntner P, Menke A, DeSalvo KB, Rabito FA, Batuman V. Continued decline in blood lead levels among adults in the United States: the National Health and Nutrition Examination Surveys. Arch Intern Med. 2005;165:2155-2161.

11. Needleman HL, Schell $A$, Bellinger D, Leviton A, Allred EN. The long-term effects of exposure to low doses of lead in childhood. An 11-year follow-up report. N Engl J Med [en línea]. 1990 [consultado 10 oct 2012];322:83-88. Disponible en: http://www.nejm.org/doi/full/10.1056/NEJM1990011132202 $03 \# \mathrm{t}=$ articleTop

12. Ornelas-Hicks $M$, Sanín-Aguirre LH, Díaz-Barriga F, Reza-López $S$, Romieu I. Evaluación de riesgo de intoxicación por plomo en la zona urbana aledaña a una fundidora en Chihuahua, México. Tecnociencia [en línea].2006 [consultado11 oct 2012]; 1:26-35. Disponible en: http://tecnociencia.uach.mx/numeros/v1n1/data/evaluacion_de_riesgo_de_intoxicacion_por_plomo.pdf

13. Pirkle JL, Kaufmann RB, Brody DJ, Hickman T, Gunter EW, Paschal D C. Exposure of the U.S. Population to Lead, 1991-1994. Environmental Health Perspectives [en línea]. 1998 [consultado 10 oct 2012];106(11):745-750. Disponible en: http://www.ncbi.nlm.nih.gov/pmc/articles/PMC1533471/pdf/ envhper00534-0101.pdf

14. Ramírez A.V. Exposición a plomo en trabajadores de fábricas informales de baterias. An Fac Med [en línea]. 2008 [consultado 11 oct 2012];69(2):104-7. Disponible en: http://www.scielo.org.pe/scielo.php?pid=S1025-5583200 8000200007 \&script $=$ sci_arttext

15. Sanín LH, González T, Romieu I, Hernández M. Acumulación de plomo en hueso y sus efectos en la salud. Salud Pública Mex [en linea]. 1998 [consultado 23 abr 2013]; 40:359-368. Disponible en: http://www.scielosp.org/ $\mathrm{pdf} / \mathrm{spm} / \mathrm{v} 40 \mathrm{n} 4 / \mathrm{Y0400409.pdf}$

16. Schwartz BS, Hu H. Adult lead exposure: time for change. Environ Health Perspect [en línea]. 2007 [consultado 10 oct 2012];115:451-454. Disponible en: http://www.ncbi.n/m.nih.gov/pmc/articles/PMC1849904/

17. Silbergeld EK. Lead in bone: implications for toxicology during pregnancy and lactation. Environ Health Perspect[en línea].1991 [consultado15 oct 2012];91:63-70. Disponible en: http://www.ncbi.n/m.nih.gov/pmc/articles/ PMC1519355/pdf/envhper00387-0065.pdf

18. Steenland K, Selevan S, Landrigan P. The mortality of lead smelter workers: an update. Am J Public Health. 1992;82:1641-1644. 